\title{
The Influence of Culture Medium Type on Cellular Phenotype of Canine Adipose Derived Stem Cells
}

\author{
Kristina M. Kiefer ${ }^{1}$, G. Elizabeth Pluhar ${ }^{1}$, Michael G. Conzemius' ${ }^{1}$, Timothy D. O'Brien² \\ ${ }^{1}$ Department of Veterinary Clinical Sciences, College of Veterinary Medicine, University of Minnesota, St. Paul, USA \\ ${ }^{2}$ Department of Veterinary Population Medicine, College of Veterinary Medicine, University of Minnesota, \\ St. Paul, USA \\ Email: kief0048@umn.edu
}

Received 23 January 2014; revised 23 February 2014; accepted 28 February 2014

Copyright (C) 2014 by authors and Scientific Research Publishing Inc.

This work is licensed under the Creative Commons Attribution International License (CC BY). http://creativecommons.org/licenses/by/4.0/

(c) (i) Open Access

\begin{abstract}
Canine adipose derived stem cells (ASCs) hold a great promise for the therapy of osteoarthritis in veterinary medicine. Current therapy is an autologous, stromal vascular fraction. Allogeneic ASCs provide many advantages, including efficient, cost-effective treatments while eliminating a surgical procedure in a diseased animal. Cultured ASCs can be expanded and characterized, allowing selection of desirable qualities. Use of allogeneic ASCs requires selection of a culture medium that provides consistent, desirable cellular products. The supplements within a medium can greatly influence cellular phenotypes. We hypothesized that medium type influenced cellular phenotype, allowing selection of a specified cellular product for clinical applications. We evaluated ASCs derived from adipose tissue of six dogs, assessing mRNA expression of proinflammatory: interleukin-1 $\beta$, cyclooxygenase-2, and anti-inflammatory mediators: tissue inhibitor metalloproteinase-2 and interleukin -1 receptor antagonist, via quantitative RT-PCR prior to, and following culture in five cell culture media: basic cell growth medium (BGM), Keratinocyte $\mathrm{N}$ acetyl-L-cysteine supplemented (KNAC) medium, Multipotent Adult Progenitor Cell (MAPC) medium, serum free medium (SFM) and xeno-free medium. Major histocompatability complex I (MHCI), major histocompatability complex II (MHCII), CD44 and CD90 immunophenotypes were assessed via flow cytometry analysis. Tri-lineage differentiation (bone, adipose and cartilage tissue) was utilized to verify multipotency. SFM and xeno-free culture conditions did not produce cell expansion sufficient to assess phenotype. ASCs prior to culture had wide variability in all mediator levels, while culturing in the remaining conditions resulted in more predictable expression levels of inflammatory mediators, with a decrease in all levels. Cultured ASCs retained expression of cell surface markers MHCI, CD44 and CD90, while decreasing MHCII expression levels. KNAC and MAPC medium conditions consistently produced tri-lineage differentation; BGM, SFM and xeno-free medium did not. Culture condition will influence phenotype of ASCs, and should be selected according to the intended therapeutic effect.
\end{abstract}




\section{Keywords}

\section{Canine; Mesenchymal Stem Cells; Adipose-Derived Stromal Cells; Culture Conditions}

\section{Introduction}

Mesenchymal stem cells have been identified and isolated from multiple adult tissues [1] [2]. They provide an ethically acceptable source of multipotent stem cells [3] [4]. They have been investigated for the therapy of many disease processes in several species. Canine adipose derived stem cells (ASCs) provide a source of mesenchymal stem cells that are potentially beneficial for the therapy of osteoarthritis in veterinary medicine and are used in clinical practice [5]-[7]. Currently, most veterinary therapy utilizes an autologous, stromal vascular fraction (SVF) generated from the patient in need of treatment [5]-[7]. Using allogeneic stem cells may provide advantages over autologous SVF, including more efficient, cost effective treatments without the need for a surgical procedure on the patient in need of therapy. Additionally, cultured stem cells can be exponentially expanded, which verified that they maintain multipotency, and selected for desirable qualities prior to use. Allogeneic stem cells have been successfully and safely used to treat other disease processes in dogs [8]-[11].

The use of allogeneic stem cells as a therapy for osteoarthritis (OA) requires the selection of a medium that provides a consistent, desirable cellular phenotype. Supplements to culture medium can greatly influence cellular phenotype, and even differentiate stem cells towards a specific cell type. This raises questions about the role of culture medium may play in altering the behavior of cultured stem cells when compared to stromal vascular fraction cells [1] [3] [4] [12]-[14].

In OA, many cytokines play a role in inflammation and pain. Thus, if treating OA with ASCs, the cellular phenotype related to expression of these cytokines is intuitively relevant. Anti-inflammatory mediators associated with a potential therapeutic effect for OA, such as tissue inhibitor of metalloproteinase-2 (TIMP-2) and interleukin 1 receptor antagonist (IL-1ra), would theoretically be beneficial, while minimal expression of proinflammatory mediators, cyclooxygenase 2 (COX-2) and interleukin 1 beta (IL-1 $\beta$ ), would be preferred [15]-[20].

Other phenotypic traits that might be important include a reduced capacity to generate a host response by minimal expression of major histocompatability complex II (MHCII) and retention of major histocompatability complex I (MCHI) [21]. Cell surface markers identified to be associated with mesenchymal stem cells, such as CD44 and CD90, should be present while hematopoeitic cell markers, CD34 and CD45, should be absent [12] [14]. Additional stem cell markers demonstrated in other stem cell types and species (CD105, CD117, CD133) would be of interest as well [12] [14]. Finally, a capacity to generate cartilage would be desirable for OA therapies.

The objective of this work was to assess the variability of cellular phenotypes suspected to play a role in the therapy of OA after exposure to different culture medium conditions. Our null hypothesis was that changes in culture medium conditions would have no influence on cellular phenotype.

\section{Materials and Methods}

\subsection{Isolation of Adipose Derived Stem Cells}

All procedures were performed in accordance with the University of Minnesota Institutional Care and Use Committee. Six, healthy, 1-year old female intact hound dogs were placed under general anesthesia and, using aseptic technique, approximately 50-gms of falciform fat was collected. Anesthetic protocol included butorphanol $(0.2 \mathrm{mg} / \mathrm{kg}$, Fort Dodge) intramuscularly as a premedication, propofol $(6 \mathrm{mg} / \mathrm{kg}$ to effect, Abbott Animal Health) intraveneously as an induction agent, and isoflurane (to effect, Vedco) gas inhalent for anesthetic maintenance. A SVF was generated from each fat sample using 0.075\% Collagenase Type I (Gibco) digestion as previously described [5] [6]. Nucleated cells were counted using a hemocytometer and each sample was aliquoted with standardized nucleated cell numbers for phenotypic assessment of SVF and subculture within each cell culture medium condition.

\subsection{Cell Culture Conditions}

Five different cell culture medium conditions were used. They were: basic cell growth medium (BGM) consist- 
ing of low glucose Dulbecco's Modified Eagle Medium $^{a}, 10 \%$ characterized fetal bovine serum ${ }^{b}$ (FBS), and 1\% antibiotics (penicillin 10,000 IU/mL, streptomycin 10,000 ug/mL, amphotericin B $25 \mathrm{ug} / \mathrm{mL}$ ) ${ }^{c}$; Keratinocyte N acetyl-L-cysteine supplemented (KNAC) medium consisting of modified MCDB153 medium (Keratinocyte$\mathrm{SFM}^{a}{ }^{a}, 2 \mathrm{mM}$ N-acetyl-L-cysteine ${ }^{d}, 0.2 \mathrm{mM} \mathrm{L-ascorbic} \mathrm{acid} \mathrm{2-phophate}{ }^{d}, 0.09 \mathrm{mM}$ calcium and human recombinant epidermal growth factor ${ }^{a}(5 \mathrm{ng} / \mathrm{mL})$, bovine pituitary extract ${ }^{a}(50 \mathrm{ug} / \mathrm{mL})$, insulin ${ }^{d}(5 \mathrm{ug} / \mathrm{mL})$, hydrocortisone $^{d}$ (74 ng/mL), 5\% FBS (Hyclone), and 1\% antibiotics ${ }^{c}$ [12]; Multipotent Adult Progenitor Cell (MAPC) medium consisting of low glucose Dulbecco's Modified Eagle Medium ${ }^{a}, 40 \%$ MCDB $^{d}, 1 \%$ L-Ascorbic Acid ${ }^{d}$, platelet derived growth factor ${ }^{e}(10 \mathrm{ng} / \mathrm{mL})$, epidermal growth factor $^{d}(10 \mathrm{ng} / \mathrm{mL})$, dexamethasone ${ }^{d}(0.5 \mathrm{uM}), 1 \%$ ITS+ liquid media supplement ${ }^{d}, 10 \%$ FBS, and $1 \%$ antibiotics [22]; serum free medium was Stempro MSC SFM Human Mesenchymal Stem Cell Culture Medium ${ }^{a}$ supplemented with $1 \%$ antibiotics and xeno-free medium consisting of StemPro MSC SFM Xeno-free medium ${ }^{a}$, supplemented with $1 \%$ antibiotics. Stromal vascular fractions from each cell line were placed into each condition at a density of 8000 nucleated cells $/ \mathrm{cm}^{2}$. Cultures were placed in incubators maintained at $37^{\circ} \mathrm{C}$ in a humidified atmosphere with $5 \% \mathrm{CO}_{2}$. After twenty-four hours, the non-adherent cells were removed by aspirating medium and rinsing the cells with $10 \mathrm{~mL}$ of phospate buffered saline. Fresh culture medium was placed on adherent cells. Culture media were changed every two days, and when $80 \%$ - 90\% confluency was reached the cells were detached using Tryple- $E^{f}$, rinsed with phospate buffered saline, and viable cells counted using the trypan blue exclusion method. Cells were then plated or frozen in liquid nitrogen for future assessment. The cell lines were expanded for a minimum of three passages when responsive to media conditions. The SVF and each subsequent cell passage in each medium condition were evaluated for multiple phenotypic properties. Population doubling time was calculated at each passage using the following formula: $P D=t \log 2 / \log$ (number of viable cells/number of cells plated), where PD is the population doubling, and $t$ is the time in culture.

\subsection{Real Time Polymerase Chain Reaction}

Proinflammatory (COX-2 and IL-1B) and anti-inflammatory (IL-1ra and TIMP-2) mediator messenger RNA levels were measured by quantitative real time polymerase chain reaction. Glyceraldehyde-2-phosphate dehydrogenase (GAPDH) was used as a housekeeping gene. The primers used for amplification, with the exception of TIMP-2 [12], were generated using the Primer 3 v 0.4.0 program and verified for gene specificity using a BLAST program ${ }^{g}$ and by gene sequencing the product of the PCR reaction ${ }^{h}$ (Table 1). Primers were synthesized by a life sciences company ${ }^{f}$. Total RNA was extracted using TRIzol Reagent ${ }^{f}$. Reverse transcription of $2 \mu \mathrm{g}$ of mRNA was performed with $2 \mu \mathrm{g}$ SuperScript III Reverse Transcriptase, $1 \mathrm{mM}$ dNTPs, 50 ng random hexamers, $20 \mathrm{mM}$ RT buffer, $5 \mathrm{mM} \mathrm{MgCl}_{2}, 100 \mathrm{mM}$ DTT, and $40 \mathrm{U}$ of RNaseOUT ${ }^{f}$ in a PTC-100 Programmable Thermal Controller ${ }^{i}$ at $65^{\circ} \mathrm{C}$ for $5 \mathrm{~min}, 4^{\circ} \mathrm{C}$ for 15 minutes, $25^{\circ} \mathrm{C}$ for 10 minutes, $50^{\circ} \mathrm{C}$ for 50 minutes, and $85^{\circ} \mathrm{C}$ for 5 minutes. Quantitative RT-PCR was performed with 6 uL Syber Green ${ }^{f}, 0.02$ ug cDNA, and $0.25 \mu \mathrm{M}$ forward and reverse primers for 40 cycles of $95^{\circ} \mathrm{C}$ for $15 \mathrm{~s}$, and $60^{\circ} \mathrm{C}$ for $60 \mathrm{~s}$ in a Mastercycler. Expression values were normalized to the housekeeping gene, GAPDH, and calculated via the $2^{=\Delta \mathrm{Ct}}$ equation [23]. mRNA expression from the SVF was compared to each of the media conditions after passage 3. Mediator expression was normalized to GAPDH and reported as percentage of GAPDH expression.

\subsection{Immunophenotype}

Cell surface marker expression evaluating immunostimulatory potential [MHCI ${ }^{l}$ (H58A), $\mathrm{MHCII}^{m}$ (555810)], mesenchymal stem cell markers [CD44 ${ }^{l}$ (BAG40A), CD90 ${ }^{l}$ (DH2A) and $\mathrm{CD}^{105^{n}}$ (9811-09)], hematopoietic stem cell markers [CD34 ${ }^{m}$ (559369), CD45 (CAD019A), CD117 $^{m}$ (555714) and CD133 ${ }^{\circ}$ (12-1331-80)] were labeled with phycoerythrin flourochrome and evaluated by flow cytometry on a BD FACSCalibur instrument ${ }^{m}$ with a $488 \mathrm{~nm}$ and $633 \mathrm{~nm}$ laser. Cells were aliquoted to $1 \times 10^{5}$ cells per cell surface marker and rinsed with FACs buffer (phosphate buffer solution with 2\% FBS) than incubated with primary antibody (MHCI, MHCII, CD44, CD45, CD90) for 30 minutes followed by secondary antibody (PE goat anti-mouse Ig, 550589) ${ }^{m}$ for 30 minutes, or a conjugated antibody marker (CD34, CD105, CD117, CD133) for 30 minutes prior to analysis.

\subsection{Multipotency}

Differentiation was initiated following the third passage in each medium condition. Adipogenic differentiation consisted of plating cells at a density of $10,000 \mathrm{cells} / \mathrm{cm}^{2}$ and exposure of cells to adipogenic differentiation me- 
Table 1. Primer sequences used in this study.

\begin{tabular}{cccc}
\hline Name & Sequence $(5 '->$ 3', forward/reverse $)$ & Expected size $(b p)$ & Reference \\
\hline COX-2 & TGAGCACAGGATTTGACCAG/CAATGTTCCAGACTCCCTTGA & 190 & This study \\
IL-1B & CTGATGGCCCTGGAAATGT/GGCTTCTTCAGCTTCTCCAA & 160 & This study \\
IL-1ra & TGCAGGTGTCCTCTCAGCTA/GAGCCTGGTCTCATCTCCAG & 214 & This study \\
TIMP-2 & ACGCGGACGTAGTGATTAGG/TTCCCGCAATGAGATACTCC & 184 & Kang, et al. \\
GAPDH & GCCAAGAGGGTCATCATCTC/CTTTGGCTAGAGGTGCCAAG & 220 & This study \\
\hline
\end{tabular}

dium consisting of low glucose $\mathrm{DMEM}^{a}$ supplemented with $5 \% \mathrm{FBS}^{b}, 0.5 \mathrm{uM}$ dexamethasone ${ }^{d}, 5 \mathrm{uM}$ insulin ${ }^{d}$, $10 \mathrm{uM}$ indomethacin ${ }^{d}$, and $0.25 \mathrm{mM}$ isobutylmethylxanthine ${ }^{d}$ for three days, then growth medium for three days, cycling for 21 days total [12] [13]. Osteogenic differentiation consisted of plating at a density of $1000 \mathrm{cells} / \mathrm{cm}^{2}$ and exposure of cells to osteogenic differentiation medium consisting of low glucose DMEM ${ }^{a}$, 5\% FBS, $50 \mathrm{nM}$

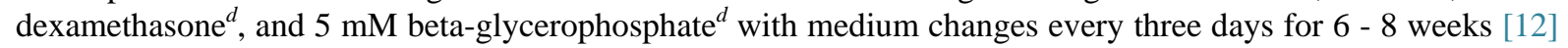
[13]. Chondrogenic differentiation consisted of pelleting 100,000 cells in $10 \mathrm{uL}$ of growth medium, and exposure of the pelleted cells to chondrogenic medium consisting of low glucose DMEM, $10 \%$ FBS, $10 \mathrm{ng} / \mathrm{mL}$ TGF- $\beta^{p}$, $50 \mathrm{uM} \mathrm{L-ascorbic} \mathrm{acid}{ }^{d}$, and $6.25 \mathrm{ug} / \mathrm{mL}$ insulin ${ }^{d}$ with medium changes every three days for 14 days [13]. Each condition was evaluated in duplicate, and compared to a negative control consisting of cells cultured at the same density and conditions with the exception of exposure to growth medium in the stead of differentiation medium.

Evaluation of adipose differentiation was accomplished with oil red O staining ${ }^{d}$, alizarin red staining ${ }^{d}$ for bone differentiation assessment, and Alcian blue staining and histological assessment was used for chondrogenic differentiation. Histologic assessment was done by one author (TDO) who was blinded to treatment group assignment. Adipose and bone differentiation is reported as a positive or negative result. Cartilage differentiation was scored on histologic assessment according to the following scale: $0=$ none (no evidence of rounded chondrocyte morphology or production of Alcian blue staining matrix), $1=$ poor ( $<10 \%$ rounded cells (ie mostly spindle cells) and minimal production of Alcian blue matrix), $2=$ fair (10\% - 25\% of cells rounded (ie many spindle cells) and small amounts of Alcian blue matrix), $3=$ good ( $25 \%-50 \%$ of cells rounded and moderate amounts of Alcian blue matrix), $4=$ excellent ( $>50 \%$ of cells rounded and abundant Alcian blue matrix).

\subsection{Statistical Analysis}

Population doubling times and quantitative RT-PCR data were analyzed using a Wilcoxon signed-rank test, with $\mathrm{p}<0.05$ considered statistically significant. All data are expressed as mean \pm standard deviation.

\section{Results}

\subsection{Isolation of Adipose Derived Stem Cells}

After harvest and processing, total nucleated cell counts were calculated for each sample. The mean \pm standard deviation total nucleated cell count was $5.9 \times 10^{6} \pm 4.65 \times 10^{6}$ cells (range: $2.065-13.35 \times 10^{6}$ cells).

\subsection{Cell Culture Conditions}

Growth of ASCs in SFM and xeno free conditions was very poor and 80\% confluency of cells was not reached in either medium condition. Therefore these conditions did not provide sufficient cell numbers for assessment of any criteria. When comparing population doubling times within a passage, BGM created significantly shorter doubling times when compared to KNAC ( $\mathrm{p}$-value $=0.028)$ and MAPC $(\mathrm{p}$-value $=0.028)$ after passage two (Figure 1).

\subsection{Real Time Polymerase Chain Reaction}

Proinflammatory cytokine gene expression significantly decreased in all of the media conditions when compared to the SVF. However, there were no statistical differences in proinflammatory marker expression between any of the media conditions (Figure 2). Anti-inflammatory mediator expression also significantly decreased in any culture conditions, with no significant differences in expression levels among media conditions (Figure 3). Not only 


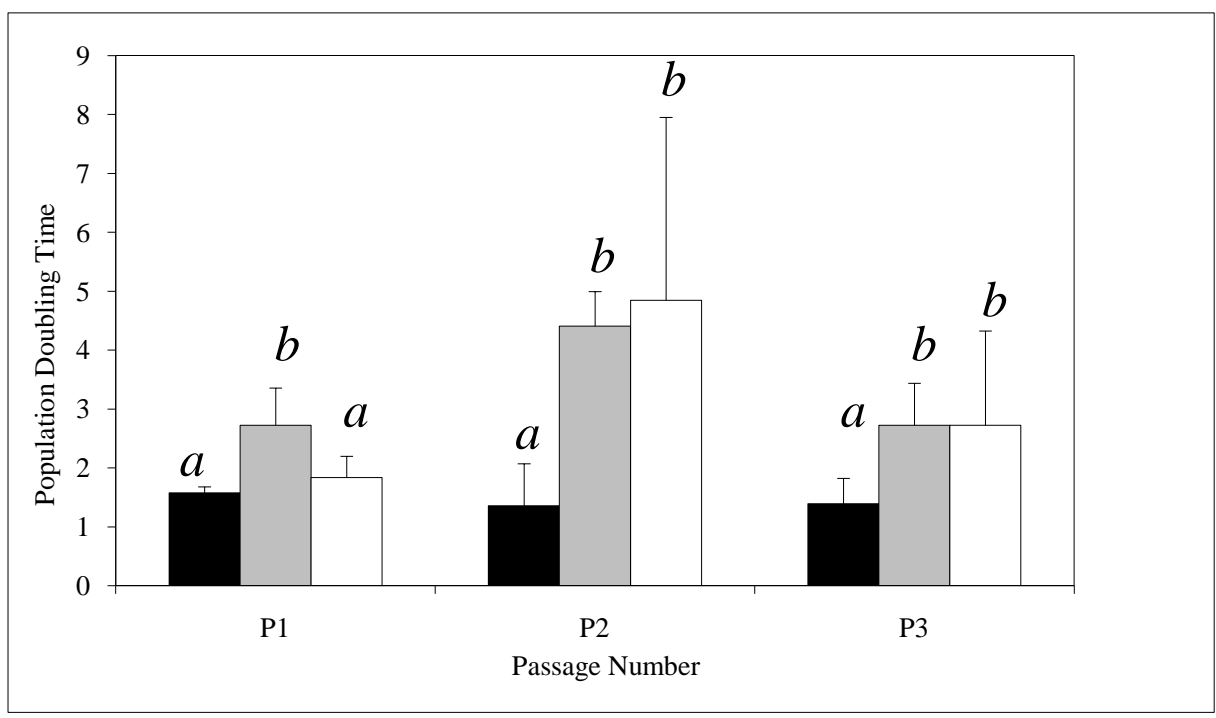

Figure 1. Average population doubling time of all cell lines $(n=6)$ in each culture medium condition at each passage (P). Time is expressed as days. Medium conditions consisted of basic growth medium (BGM; black), Keratinocyte N-acetylcholine medium (KNAC; grey), multipotent adult progenitor cell medium (MAPC; white). Conditions with a different letter indicate significant differences in population doubling times within a passage; not between passages.

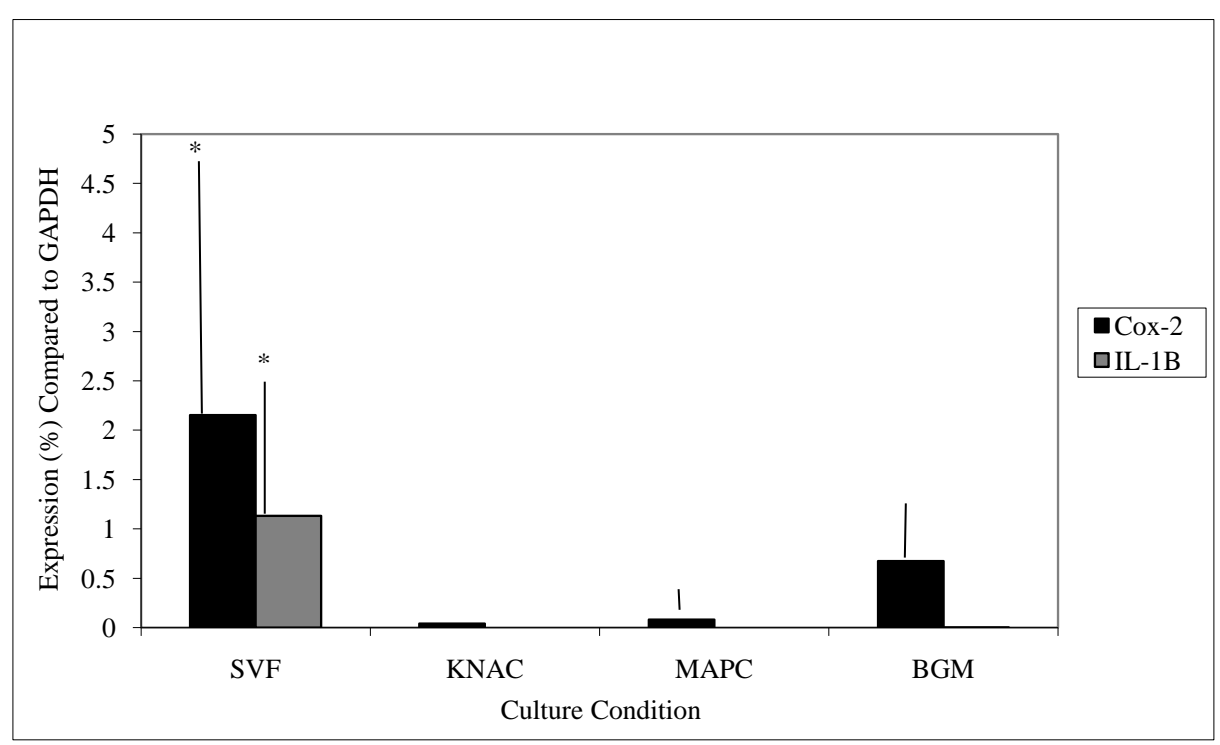

Figure 2. Gene expression of proinflammatory cytokines, COX-2 and IL-1B, at initial harvest (SVF), and at passage 3 following exposure to each culture condition consisting of basic growth medium (BGM), keratinocyte $\mathrm{N}$-acetylcholine medium (KNAC), multipotent adult progenitor cell medium (MAPC). Cytokine gene expression was measured with quantitative RT-PCR, and expression values were normalized to the housekeeping gene, GAPDH, and given as a percentage of expression. An asterisk (*) indicates a significant difference between SVF and passage 3; there were no statistically significant differences among media conditions.

SVFs expressed significantly higher levels of all mediators, but variation about the mean was dramatically higher.

\subsection{Immunophenotype}

MHCI expression was present in all conditions. MHCII was expressed in a small population of SVF cells (10.5\%) and then significantly decreased once cells were cultured (Figure 4). All medium conditions retained 


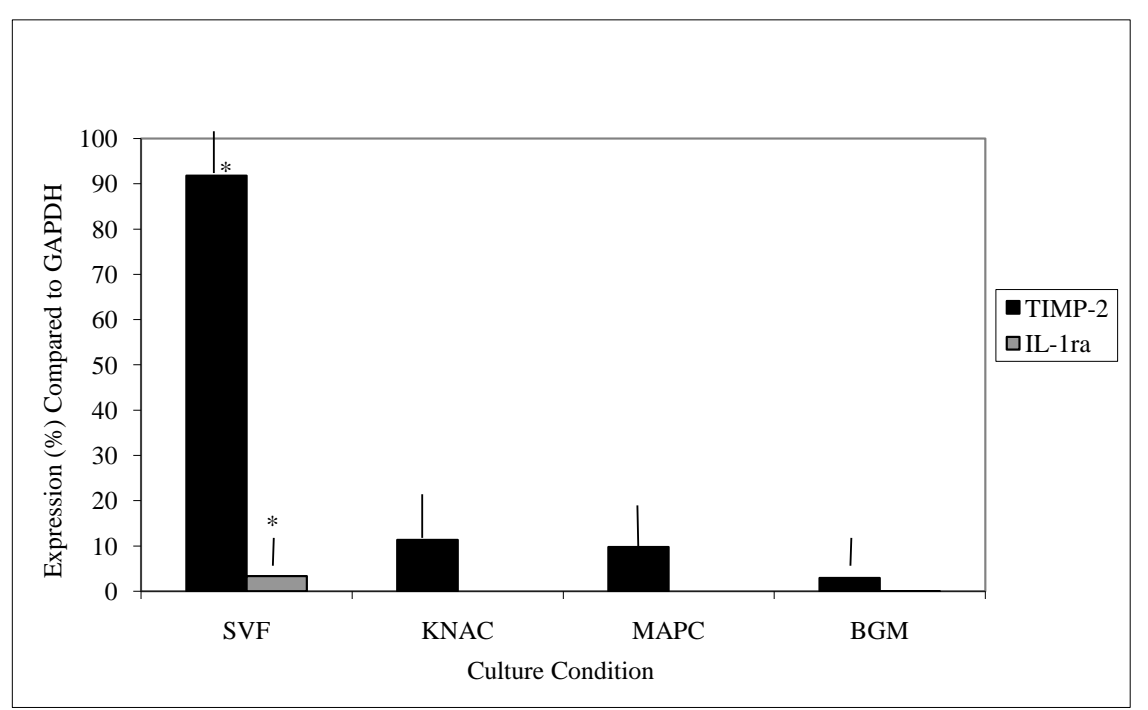

Figure 3. Gene expression for anti-inflammatory mediators, TIMP-2 and IL-1ra, at initial harvest (SVF), and at passage 3 following exposure to each culture condition. Mediator gene expression was measured with quantitative RT- PCR and expression values were normalized to the housekeeping gene, GAPDH, and reported as a percentage of expression. An asterisk $(*)$ indicates a significant difference between SVF and passage 3; there were no statistically significant differences among media conditions.
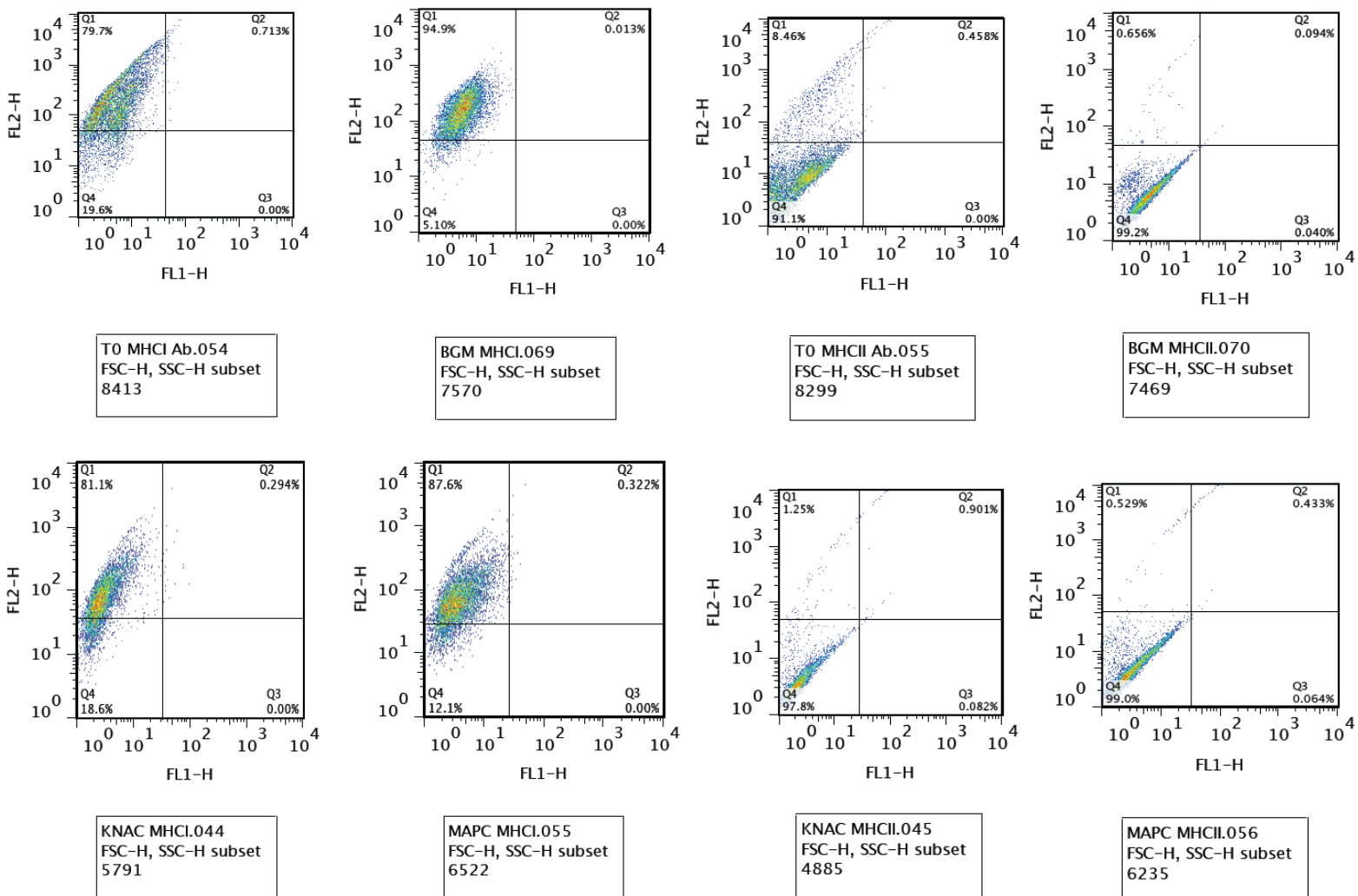

Figure 4. Flow cytometry analysis of MHCI and MHCII expression levels in SVF (T0) and each medium condition after three passages. Cell populations contained within the lower left quadrant indicate no expression of the marker of interest. Cell populations contained within the upper left quadrant indicate positive expression of the marker of interest. The total percent of cells falling within each quadrant is listed within that quadrant. Cultured cells retained MHCI when compared with SVF, while decreasing expression of MHCII. 
cell populations expressing CD44 and CD90, although BGM conditions retained a larger percentage of CD90expressing cells. A small percentage of cells (9.5\%) expressed CD105 in the SVF, but CD105 expression was lost after culture in any media condition. Similarly, CD34 (44.7\%) and CD45 (17.05\%) expression was found in the SVF, but not in cell populations after culture. CD117 and CD133 were not expressed by any population of cells.

\subsection{Multipotency}

All culture conditions that enabled cell expansion also allowed tissue differentiation, but cells in BGM medium did not generate adipocytes or osteocytes. KNAC and MAPC both allowed tri-lineage differentiation (Table 2).

\section{Discussion}

Isolation of canine adipose derived stem cells was successful as previously reported [5] [7] [12]-[14], but provided variable yields of nucleated cells, indicating that certain individuals may provide more SVF treatments than others. Determining the characteristics to identify individuals that might provide greater yields was beyond the scope of this study. A greater nucleated cell yield does not necessarily provide a greater ASC yield, as the method for counting nucleated cells does not distinguish cell type.

SVF gene expression of pro and anti-inflammatory mediators consistently decreased after 3 passages in cell culture and achieved similar levels in all culture conditions. This suggests that either the majority of the expression of the mediators is from cells that are lost from the SVF in cell culture and are not present in the ASC or that each of the culture conditions resulted in similar changes in gene expression in cells comprising the ASC. If the decrease in proinflammatory cytokine gene expression after passage is advantageous, then the decrease in anti-inflammatory mediators may be disadvantageous. Isolation of the specific cell type(s) from the SVF that result in the preferential expression of either pro or anti-inflammatory mediators would be useful.

Table 2. Differentiation potential for each cell line in each medium condition consisting of basic growth medium (BGM), Keratinocyte $\mathrm{N}$-acetylcholine medium (KNAC), multipotent adult progenitor cell medium (MAPC). Cartilage differentiation (chondrocytes) is graded as $0=$ none, 1 = poor, 2 = fair, 3 = good, 4 = excellent, and adipocyte (adipo) and osteocyte (osteo) conditions are listed as positive or negative differentiation. A negative score for cartilage differentiation indicates no generation of cell pellet for histologic analysis.

\begin{tabular}{|c|c|c|c|c|}
\hline Condition & Line & Adipo & Osteo & Chondrocytes \\
\hline \multirow[t]{6}{*}{ BGM } & 1 & - & - & 1 \\
\hline & 2 & - & - & 1 \\
\hline & 3 & - & - & 1 \\
\hline & 4 & - & - & - \\
\hline & 5 & - & - & - \\
\hline & 6 & - & - & - \\
\hline \multirow[t]{6}{*}{ MAPC } & 1 & + & + & - \\
\hline & 2 & + & + & 3 \\
\hline & 3 & + & contaminated & - \\
\hline & 4 & + & + & - \\
\hline & 5 & + & + & 3 \\
\hline & 6 & + & + & - \\
\hline \multirow[t]{6}{*}{ KNAC } & 1 & + & + & - \\
\hline & 2 & + & + & 3 \\
\hline & 3 & + & contaminated & 2 \\
\hline & 4 & + & + & 3 \\
\hline & 5 & + & + & - \\
\hline & 6 & + & + & - \\
\hline
\end{tabular}


Stromal vascular fractions contain a heterogeneous population of cells, including red blood cells, leukocytes, adipocytes, and a small population of ASCs [24]. This may be an explanation for the variations in expression of surface markers and mediator expression in SVF populations. Of these cell populations, only the progenitor and stromal cells should be capable of self-renewal, eliminating much of the heterogeneity of cell type by culturing. This is a possible explanation for the apparent loss of cell populations in the FACS data following cell culture, specifically the CD34+, CD44-, CD45+, CD105+, and MHCII+ cells that were seen in the SVF. Alternatively, the change in expression may be due to a change in phenotype of a persistent population of cells. Similar loss of mediator expression in passaged cells may be explained by the same mechanisms. Culturing the SVF appears to generate cells of a more uniform phenotype, regardless of the donor or medium type.

Identifying individual cell populations as ASCs is difficult, as surface markers commonly used to identify ASCs are not specific for that population. CD44, CD90 and CD105 are considered to be markers of mesenchymal stem/stromal cells but may be found in many differentiated mesenchymal cells across many species. CD105 was not expressed on canine ASCs in a previous study; a finding that was corroborated in our study [14]. It is interesting to note that a small percentage of cells appeared to express this marker prior to culture. The presence of some cells expressing hematopoetic stem cell markers (CD45, CD34) is not surprising in the SVF due to the heterogeneous nature of this cell population.

The variable nature of SVF makes the concept of culturing cells to generate a consistent phenotype and cell population appealing. The cultured cells could be used either as an autologous or allogeneic product. The ability to expand the cells in culture would also facilitate the treatment of multiple individuals with a single, characterized product. In our study, SFM and xeno-free medium conditions were unsuccessful for expanding canine cell populations, in spite of success in other species. This is not surprising given the variability in stem cell characteristics and qualities between species or even source. A recent study identified an alternate serum free medium supplemented with a serum substitute that was successful in providing adequate proliferation in canines [25]. The elimination of fetal bovine serum from the culture medium would be ideal, due to the highly variable content of this material, and therefore the potential for variable phenotype of cells in the medium, and the fact that it is a xenobiotic material that may induce an immune reaction. Therefore, even though the SFM tested in this study was not found suitable for expansion of canine ASCs, the pursuit of SFM for this purpose should not be abandoned, and appears possible with the correct conditions. Canine serum could be considered an alternative and evaluated for the purposes of growth expansion, but will likely still carry a high variability in product content.

The immunogenic potential of allogeneic ASCs in transplants has been raised, but in studies thus far, appears to be feasible for clinical use [8] [12] [26] [27]. An immune response relies in part, upon the expression of MHCII on the cell surface, but the absence of MHCI on cell surfaces will also elicit an immune response [21]. Our study demonstrated that the level of MHCII is diminished following culture in all populations of cells, while expression of MHCI in low levels are retained. Thus, culturing does not appear to increase an immune response.

It is interesting to note that cells cultured in BGM had efficient population doublings (Figure 1) and retained ASC markers CD44 and CD90, but had a poor capacity to differentiate (Table 2). In contrast, KNAC and MAPC cultured cells had longer doubling times but retained the capacity to differentiate into multiple lineages. Differences in the expansion medium composition may explain these differences by promoting alternate phenotypes among the cells or by selecting for cells with differing characteristics. Notably, the differences between phenotypes of cells cultured in KNAC and MAPC were minimal.

The low number of cell lines investigated is a limitation of this study. In addition, it is important to note that variation in the source and supply of reagents, particularly fetal bovine serum, may alter phenotype. The FBS used in this study was from a single lot to minimize variability. A further study investigating variability of phenotypes when exposed to different lots and sources of FBS would be prudent. Alternatively, a standard set of phenotypic characteristics selected as ideal for the therapeutic application could be utilized to establish an acceptable phenotype against which all new lots of FBS be measured.

\section{Conclusion}

Culture of canine adipose derived stem cells led to an altered phenotypic profile compared to SVF. The type of culture medium had an effect on phenotype, so we reject our null hypothesis that change in culture medium conditions would have no influence on cellular phenotype. However, with the exception of the capacity for the cells to differentiate, the differences were minimal. The selection of culture medium should be made based upon 
the desired therapeutic application and the qualities’ pertinent for that particular use. Allogeneic stem cell therapy appears in feasible warrants in vivo investigation.

\section{Acknowledgements}

The authors would like to thank Rich Evans for support in statistical analysis of data and Jamie Modiano for resources in flow cytometry analysis.

\section{References}

[1] Kang, B.J., Ryu, H.H., Park, S.S., et al. (2012) Comparing the Osteogenic Potential of Canine Mesenchymal Stem Cells Derived from Adipose Tissues, Bone Marrow, Umbilical Cord Blood, and Wharton's Jelly for Treating Bone Defects. Journal of Veterinary Science, 13, 299-310. http://dx.doi.org/10.4142/jvs.2012.13.3.299

[2] Kisiel, A.H., McDuffee, L.A., Masaoud, E., Bailey, T.R., Esparza Gonzalez, B.P. and Nino-Fong, R. (2012) Isolation, Characterization, and in Vitro Proliferation of Canine Mesenchymal Stem Cells Derived from Bone Marrow, Adipose Tissue, Muscle, and Periosteum. American Journal of Veterinary Research, 73, 1305-1317. http://dx.doi.org/10.2460/ajvr.73.8.1305

[3] Csaki, C., Matis, U., Mobasheri, A., Ye, H. and Shakibaei, M. (2007) Chondrogenesis, Osteogenesis and Adipogenesis of Canine Mesenchymal Stem Cells: A Biochemical, Morphological and Ultrastructural Study. Histochemistry and Cell Biology, 128, 507-520. http://dx.doi.org/10.1007/s00418-007-0337-z

[4] Reich, C.M., Raabe, O., Wenisch, S., Bridger, P.S., Kramer, M. and Arnhold, S. (2012) Isolation, Culture and Chondrogenic Differentiation of Canine Adipose Tissue- and Bone Marrow-Derived Mesenchymal Stem Cells-A Comparative Study. Veterinary Research Communications, 36, 139-148. http://dx.doi.org/10.1007/s11259-012-9523-0

[5] Black, L.L., Gaynor, J., Adams, C., et al. (2008) Effect of Intraarticular Injection of Autologous Adipose-Derived Mesenchymal Stem and Regenerative Cells on Clinical Signs of Chronic Osteoarthritis of the Elbow Joint in Dogs. Veterinary Therapeutics, 9, 192-200.

[6] Black, L.L., Gaynor, J., Gahring, D., et al. (2007) Effect of Adipose-Derived Mesenchymal Stem and Regenerative Cells on Lameness in Dogs with Chronic Osteoarthritis of the Coxofemoral Joints: A Randomized, Double-Blinded, Multicenter, Controlled Trial. Veterinary Therapeutics, 8, 272-284.

[7] Guercio, A., Di Marco, P., Casella, S., et al. (2012) Production of Canine Mesenchymal Stem Cells from Adipose Tissue and Their Application in Dogs with Chronic Osteoarthritis of the Humeroradial Joints. Cell Biology International, 36, 189-194. http://dx.doi.org/10.1042/CBI20110304

[8] Arinzeh, T.L., Peter, S.J., Archambault, M.P., et al. (2003) Allogeneic Mesenchymal Stem Cells Regenerate Bone in a Critical-Sized Canine Segmental Defect. The Journal of Bone \& Joint Surgery of America, 85-A, 1927-1935.

[9] Park, S.S., Lee, Y.J., Lee, S.H., et al. (2012) Functional Recovery after Spinal Cord Injury in Dogs Treated with a Combination of Matrigel and Neural-Induced Adipose-Derived Mesenchymal Stem Cells. Cytotherapy, 14, 584-597. http://dx.doi.org/10.3109/14653249.2012.658913

[10] Perin, E.C., Silva, G.V., Assad, J.A., et al. (2008) Comparison of Intracoronary and Transendocardial Delivery of Allogeneic Mesenchymal Cells in a Canine Model of Acute Myocardial Infarction. Journal of Molecular and Cellular Cardiology, 44, 486-495. http://dx.doi.org/10.1016/j.yjmcc.2007.09.012

[11] Ryu, H.H., Lim, J.H., Byeon, Y.E., et al. (2009) Functional Recovery and Neural Differentiation after Transplantation of Allogenic Adipose-Derived Stem Cells in a Canine Model of Acute Spinal Cord Injury. Journal of Veterinary Science, 10, 273-284. http://dx.doi.org/10.4142/jvs.2009.10.4.273

[12] Kang, J.W., Kang, K.S., Koo, H.C., Park, J.R., Choi, E.W. and Park, Y.H. (2008) Soluble Factors-Mediated Immunomodulatory Effects of Canine Adipose Tissue-Derived Mesenchymal Stem Cells. Stem Cells and Development, 17, 681-693. http://dx.doi.org/10.1089/scd.2007.0153

[13] Neupane, M., Chang, C.C., Kiupel, M. and Yuzbasiyan-Gurkan, V. (2008) Isolation and Characterization of Canine Adipose-Derived Mesenchymal Stem Cells. Tissue Engineering Part A, 14, 1007-1015.

http://dx.doi.org/10.1089/tea.2007.0207

[14] Vieira, N.M., Brandalise, V., Zucconi, E., Secco, M., Strauss, B.E. and Zatz, M. (2010) Isolation, Characterization, and Differentiation Potential of Canine Adipose-Derived Stem Cells. Cell Transplantation, 19, 279-289. http://dx.doi.org/10.3727/096368909X481764

[15] Alam, M.R., Ji, J.R., Kim, M.S. and Kim, N.S. (2011) Biomarkers for Identifying the Early Phases of Osteoarthritis Secondary to Medial Patellar Luxation in Dogs. Journal of Veterinary Science, 12, 273-280. http://dx.doi.org/10.1002/art.1780390914

[16] Caron, J.P., Fernandes, J.C., Martel-Pelletier, J., et al. (1996) Chondroprotective Effect of Intraarticular Injections of 
Interleukin-1 Receptor Antagonist in Experimental Osteoarthritis. Suppression of Collagenase-1 Expression. Arthritis \& Rheumatology, 39, 1535-1544. http://dx.doi.org/10.1002/art.1780390914

[17] Clements, D.N., Carter, S.D., Innes, J.F., Ollier, W.E. and Day, P.J. (2006) Analysis of Normal and Osteoarthritic Canine Cartilage mRNA Expression by Quantitative Polymerase Chain Reaction. Arthritis Research \& Therapy, 8, R158. http://dx.doi.org/10.1186/ar2053

[18] Clements, D.N., Fitzpatrick, N., Carter, S.D. and Day, P.J. (2009) Cartilage Gene Expression Correlates with Radiographic Severity of Canine Elbow Osteoarthritis. The Veterinary Journal, 179, 211-218. http://dx.doi.org/10.1016/j.tvjl.2007.08.027

[19] Fernandes, J.C., Martel-Pelletier, J. and Pelletier, J.P. (2002) The Role of Cytokines in Osteoarthritis Pathophysiology. Biorheology, 39, 237-246.

[20] Fernandes, J., Tardif, G., Martel-Pelletier, J., et al. (1999) In Vivo Transfer of Interleukin-1 Receptor Antagonist Gene in Osteoarthritic Rabbit Knee Joints: Prevention of Osteoarthritis Progression. American Journal of Pathology, 154, 1159-1169. http://dx.doi.org/10.1016/S0002-9440(10)65368-0

[21] Ljunggren, H.G. and Karre, K. (1990) In Search of the "Missing Self”: MHC Molecules and NK Cell Recognition. Immunology Today, 11, 237-244. http://dx.doi.org/10.1016/0167-5699(90)90097-S

[22] Jiang, Y., Vaessen, B., Lenvik, T., Blackstad, M., Reyes, M. and Verfaillie, C.M. (2002) Multipotent Progenitor Cells Can Be Isolated from Postnatal Murine Bone Marrow, Muscle, and Brain. Experimental Hematology, 30, 896-904. http://dx.doi.org/10.1016/S0301-472X(02)00869-X

[23] Livak, K.J. and Schmittgen, T.D. (2001) Analysis of Relative Gene Expression Data Using Real-Time Quantitative PCR and the 2(-Delta Delta C(T)) Method. Methods, 25, 402-408. http://dx.doi.org/10.1006/meth.2001.1262

[24] Riordan, N.H., Ichim, T.E., Min, W.P., et al. (2009) Non-Expanded Adipose Stromal Vascular Fraction Cell Therapy for Multiple Sclerosis. Journal of Translational Medicine, 7, 29.

[25] Schwarz, C., Leicht, U., Rothe, C., et al. (2012) Effects of Different Media on Proliferation and Differentiation Capacity of Canine, Equine and Porcine Adipose Derived Stem Cells. Research in Veterinary Science, 93, 457-462. http://dx.doi.org/10.1016/j.rvsc.2011.08.010

[26] Gotherstrom, C., Ringden, O., Tammik, C., Zetterberg, E., Westgren, M. and Le Blanc, K. (2004) Immunologic Properties of Human Fetal Mesenchymal Stem Cells. American Journal of Obstetrics \& Gynecology, 190, 239-245. http://dx.doi.org/10.1016/j.ajog.2003.07.022

[27] Le Blanc, K., Tammik, C., Rosendahl, K., Zetterberg, E. and Ringden, O. (2003) HLA Expression and Immunologic Properties of Differentiated and Undifferentiated Mesenchymal Stem Cells. Experimental Hematology, 31, 890-896. http://dx.doi.org/10.1016/S0301-472X(03)00110-3

\section{Appendix}

$a$ Gibco, Life Technologies, Grand Island, New York

$b$ Hyclone, Thermo Fischer Scientific, Minneapolis, MN

c Mediatech Inc, Corning, NY

$d$ Sigma-Aldrich, St. Louis, MO

$e$ R\&D Systems, Minneapolis, MN

$f$ Invitrogen, Life Technologies, Grand Island, New York

$g$ BLAST, National Center for Biotechnology Information, National Institutes of Health, Betheseda, MD. Available at blast.ncbi.nlm.nih.gov/

$h$ Biomedical Genomics Center, University of Minnesota Core Factility, Minneapolis, MN

i MJ Research, Inc, St. Bruno, Canada

j Bio-Rad Laboratories, Hercules, CA

$k$ Eppendorf, Hamburg, Germany

$l$ VMRD inc, Pullman, WA

$m$ BD Biosciences, San Jose, California

$n$ Southern Biotech, Birmingham, AL

$o$ eBiosciences, San Diego, CA

$p$ Peprotech, Inc., Rocky Hill, NJ 